\title{
Local Nonlinear Model Matching: From Linearity to Nonlinearity*
}

\author{
H. J. C. HUIJBERTS $\dagger$ and H. NIJMEIJER $\dagger$
}

\begin{abstract}
Under the assumption that the model is decouplable and under generic assumptions on the plant, the nonlinear model matching problem is locally solvable if and only if it is solvable for the linearization of plant and model around an equilibrium point
\end{abstract}

Key Words-Nonlinear control systems; control systems synthesis; linearization techniques.

\begin{abstract}
The model matching problem consists of designing a compensator for a given system, called the plant, in such a way that the resulting input-output behaviour matches that of a prespecified model. In this paper a local solution of the nonlinear model matching problem is given for the case that the model is decouplable by static state feedback. The main theorem states that under generic conditions on the plant the problem is solvable around an equilibrium point if and only if it is solvable for the linearization of plant and model. The generic conditions are identified. They naturally appear in the solution of the dynamic input-output decoupling problem for the plant. The theory is illustrated by means of two examples.
\end{abstract}

\section{INTRODUCTION}

IN THIS paper we study the so called model matching problem (MMP) for nonlinear systems. That is, given a nonlinear control system, to be referred to as the plant $P$, together with another nonlinear system, to be called the model $M$, the question is whether or not it is possible to design a suitable precompensator for the plant such that the input-output behaviour of the precompensated plant matches that of the given model $M$. The idea is that in case a positive answer to this question exists, one is able to follow all the desired input-output trajectories of the model with those of the plant with precompensator.

The model matching problem has received a lot of attention during the last decades and considerable progress towards a characterization of necessary and sufficient conditions for its

\footnotetext{
* Received 11 April 1989; revised 8 November 1989; received in final form 28 November 1989 . The original version of this paper was not presented at any IFAC meeting. This paper was recommended for publication in revised form by Associate Editor V. I. Utkin under the direction of Editor H. Kwakernaak.

+ Department of Applied Mathematics, University of Twente, P.O. Box 217, 7500 AE Enschede, The Netherlands.
}

solvability has been made. Let us first quickly review the main contributions on the problem under consideration. The linear model matching problem was completely solved in the early seventies, first by Moore and Silverman (1972), using Silverman's structure algorithm (Silverman, 1969) and then by Morse (1973) using a geometric setting. Later it was realized (Morse, 1976; Emre and Hautus, 1980) that the model matching problem is equivalent to a certain disturbance decoupling problem with disturbance measurements. Finally, Malabre (1982) stated equivalent solvability conditions in terms of certain structures at infinity.

A first step towards the solution of the nonlinear model matching problem was taken in (Isidori, 1985a), where sufficient conditions were given in case the model is a linear system. A very important and innovative contribution for the general nonlinear MMP was given in the paper of Di Benedetto and Isidori (1986). In this paper a detailed problem formulation and sufficient conditions for the existence of a local solution were given. Moreover under some rather restrictive assumptions on the model and plant necessary and sufficient conditions for the existence of a local solution were given. Essentially the approach taken in Di Benedetto and Isidori (1986) was very much inspired by the recently developed nonlinear differential geometric theory, see for instance the survey by Isidori (1985b), and it forms a direct generalization of the geometric linear theory (Morse, 1976; Emre and Hautus, 1980; Malabre, 1982). Following the approach of $\mathrm{Di}$ Benedetto and Isidori (1986) other partial solutions have appeared (Di Benedetto, 1988a, 1988b; Huijberts, 1989a). To what extent the machinery of Di Benedetto and Isidori (1986) allows for a 
complete answer to the problem is still an unsolved problem.

In the present paper we take a different approach for studying the nonlinear MMP. Throughout we assume the model to be input-output decouplable by static state feedback (see Section 4 for a precise definition). Although this assumption is certainly restrictive, it can be argued that in practical circumstances it is often desirable. Given the forementioned assumption an obvious condition for the local solvability of the MMP is that the plant $P$ is input-output decouplable by adding a dynamic precompensator. The dynamic input-output decoupling problem has been solved by Descusse and Moog (1985) and later on in a slightly different way by Nijmeijer and Respondek (1988). The solvability of the MMP now may be checked by using a minimal order decoupling precompensator for the plant $P$.

The present method has an important feature that it is completely analytic and avoids concepts such as controlled invariant distributions and so on, as were used in Di Benedetto and Isidori (1986). All computations are straightforward in terms of the data of $P$ and $M$ and may be executed by formula manipulation, using e.g. MACSYMA, MAPLE or REDUCE. There is however another essential aspect of our solution we want to emphasize. Namely, we show that under generic conditions-a mathematical phrasing of almost always-the nonlinear MMP is solvable around equilibria of model $M$ and plant $P$ if and only if the corresponding linear MMP is solvable for the linearized model $L M$ and plant $L P$. In our opinion this has important practical implications, in that in engineering practice one often studies a specific control problem by addressing the problem on the linearization around a given working point. The result given here may be viewed as an a posteriori justification of this methodology. In this way the paper fits in the philosophy developed by Gras and Nijmeijer (1989), where the relation between the input-output decoupling problem for a nonlinear system and its linearization around a working point have been investigated. In fact, as a byproduct we extend the results of that paper by discussing the dynamic inputoutput decoupling problem under linearization.

Finally, after having submitted this paper, we received a very interesting preprint by Moog et al. (1989) in which, using differential algebraic tools, a solution of the nonlinear MMP is given that generalizes the results of Moore and Silverman (1972) to nonlinear systems, and which apparently matches with the sufficiency results given here.
The paper is organized as follows. In the following section we will formulate the linear model matching problem and give conditions for the solvability of this problem. In Section 3 a formulation of the nonlinear model matching problem will be given along the lines of $\mathrm{Di}$ Benedetto and Isidori (1986). Furthermore we will, after we have treated the results of $\mathrm{Di}$ Benedetto and Isidori (1986), state our main theorem. This main theorem holds true under generic conditions on the plant $P$. The conditions naturally appear in the solution of the input-output decoupling problem for the plant $P$. This solution will be given in Section 4 . Moreover, we will give some results on the minimal order linear input-output decoupling problem in Section 4. In Section 5 the main theorem will be proved and a characterization of models that can be matched starting from a specific plant will be given. In Section 6 the theory will be illustrated by means of two examples. The first one, which is borrowed from Di Benedetto and Isidori (1986), see also Di Benedetto (1988a), illustrates the constructiveness of our method. The second one, which is taken from De Luca and Ulivi (1988), illustrates our method in case the plant is a voltage frequency-controlled induction motor. In Section 7 , final conclusions will be drawn.

\section{THE LINEAR MODEL MATCHING PROBLEM}

Consider a linear plant $P$, described by equations of the form:

$$
P\left\{\begin{array}{l}
\dot{x}=A x+B u \\
y=C x
\end{array}\right.
$$

with state $x \in \mathbb{R}^{n}$, input $u \in \mathbb{R}^{m}$, output $y \in \mathbb{R}^{m}$ and matrices $A, B, C$ of appropriate dimensions. Also, let a linear model $M$ be given, which is described by the equations:

$$
M\left\{\begin{array}{l}
\dot{x}^{M}=A^{M} x^{M}+B^{M} u^{M} \\
y^{M}=C^{M} x^{M}
\end{array}\right.
$$

with state $x^{M} \in \mathbb{R}^{n_{M}}$, input $u^{M} \in \mathbb{R}^{m}$, output $y^{M} \in \mathbb{R}^{m}$ and matrices $A^{M}, B^{M}, C^{M}$ of appropriate dimensions.

The compensator $Q$ used to control $P$ is a linear system described by equations of the form:

$$
Q\left\{\begin{aligned}
\dot{x}_{c} & =K x_{c}+L x+M u^{M} \\
u & =F x_{c}+G x+H u^{M}
\end{aligned}\right.
$$

with state $x_{c} \in \mathbb{R}^{v}$ and matrices $K, L, M, F, G$, $H$ of appropriate dimensions. The composition of (1) and (3) is denoted by $P \circ Q$.

Then the model matching problem consists of finding (if possible) an integer $v$ and a 
compensator $Q$ of the form (3) such that the transfer matrices of $M$ and $P \circ Q$ coincide, so that one is able to design a precompensator $Q$ for the plant $P$ such that the input-output behaviour of $P \circ Q$ matches that of the model $M$.

A way to solve this problem is as follows. Define the matrices

$$
\begin{gathered}
A^{E}=\left[\begin{array}{cc}
A & 0 \\
0 & A^{M}
\end{array}\right] \quad B^{E}=\left[\begin{array}{cc}
B & 0 \\
0 & B^{M}
\end{array}\right] \\
C^{E}=\left[\begin{array}{ll}
C & -C^{M}
\end{array}\right] .
\end{gathered}
$$

Theorem 1. (Morse, 1973). The model matching problem is solvable if and only if

$$
\operatorname{Im}\left[\begin{array}{c}
0 \\
B^{M}
\end{array}\right] \subset \operatorname{Im}\left[\begin{array}{c}
B \\
0
\end{array}\right]+\mathscr{V}^{*}
$$

where $\mathscr{V}^{*}$ is the maximal $\left(A^{E}, B^{E}\right)$-invariant subspace in $\operatorname{Ker} C^{E}$.

The above problem also admits a direct interpretation in terms of the structure at infinity, cf. Malabre (1982).

Theorem 2. The model matching problem is solvable if and only if the system (1) and the system defined by the matrices $\left(A^{E}, B^{E}, C^{E}\right)$ of (4) have the same structure at infinity.

\section{THE NONLINEAR MODEL MATCHING PROBLEM}

The definition of the nonlinear model matching problem will follow the same lines as the definition of the linear model matching problem. We employ the definition of $\mathrm{Di}$ Benedetto and Isidori (1986).

Consider a nonlinear plant $P$, described by equations of the form:

$$
P\left\{\begin{array}{l}
\dot{x}=f(x)+\sum_{i=1}^{m} u_{i} g_{i}(x) \\
y_{i}=h_{i}(x) \quad(i \in\{1, \ldots, m\})
\end{array}\right.
$$

with state $x \in \mathscr{X}$, an open neighbourhood in $\mathbb{R}^{n}$, inputs $u_{i} \in \mathbb{R}$, outputs $y_{i} \in \mathbb{R}, f, g_{1}, \ldots, g_{m}$ are real analytic vector fields on $\mathbb{R}^{n}$ and $h_{1}, \ldots, h_{m}$ are real analytic functions.

Furthermore, let a nonlinear model $M$ be given, which is described by the equations:

$$
M\left\{\begin{array}{l}
\dot{x}^{M}=f^{M}\left(x^{M}\right)+\sum_{i=1}^{m} u_{i}^{M} g_{i}^{M}\left(x^{M}\right) \\
y_{i}^{M}=h_{i}^{M}\left(x^{M}\right) \quad(i \in\{1, \ldots, m\})
\end{array}\right.
$$

with state $x^{M} \in \mathscr{X}^{M}$, an open neighbourhood in $\mathbb{R}^{n_{M}}$, inputs $u_{i}^{M} \in \mathbb{R}$, outputs $y_{i}^{M} \in \mathbb{R}$, $f^{M}, g_{1}^{M}, \ldots, g_{m}^{M}$ are real analytic vector fields on $\mathbb{R}^{n_{M}}$ and $h_{1}^{M}, \ldots, h_{m}^{M}$ are real analytic functions.

The compensator $Q$ used to control $P$ is a nonlinear system described by equations of the form:

$$
Q\left\{\begin{array}{c}
\dot{x}_{c}=a\left(x_{c}, x\right)+b\left(x_{c}, x\right) u^{M} \\
u=c\left(x_{c}, x\right)+d\left(x_{c}, x\right) u^{M}
\end{array}\right.
$$

with state $x_{c} \in \mathscr{X}_{c}$, an open neighbourhood in $\mathbb{R}^{v}$, and real analytic $a, b, c, d$. The composition of (6) and (8) is again denoted by $P \circ Q$.

In Section 2 we saw that in the case of linear systems the objective of model matching is to design a compensator $Q$ such as to impose the coincidence between the transfer matrix of $M$ and that of $P \circ Q$. In the case of nonlinear systems, where the input-output behaviour may be described in terms of Volterra series expansions, the object of model matching is to impose the coincidence of corresponding Volterra kernels.

Recall that the output $y(t)=\left(y_{1}(t), \ldots\right.$, $y_{m}(t)$ ) of a nonlinear system of the form (6) has a Volterra series expansion of the form [see e.g. Isidori (1985b)]

$$
\begin{aligned}
y(t)= & w_{0}\left(t, x_{0}\right)+\sum_{i=1}^{m} \int_{0}^{t} w_{i}\left(t, \tau_{1}, x_{0}\right) u_{i}\left(\tau_{1}\right) \mathrm{d} \tau_{1} \\
& +\sum_{i_{1}, i_{2}=1}^{m} \int_{0}^{t} \int_{0}^{\tau_{1}} w_{i_{1} i_{2}}\left(t, \tau_{1}, \tau_{2}, x_{0}\right) u_{i_{1}}\left(\tau_{1}\right) \\
& \times u_{i_{2}}\left(\tau_{2}\right) \mathrm{d} \tau_{1} \mathrm{~d} \tau_{2}+\cdots
\end{aligned}
$$

where $x_{0}$ is the initial state at time $t=0$.

Let $w_{j_{1} \ldots j_{i}}^{M}\left(t, \tau_{1}, \ldots, \tau_{i}, x_{0}^{M}\right)$ denote the $\left(j_{1}, \ldots, j_{i}\right)$-th kernel of model $M$ and similarly $\boldsymbol{w}_{j_{1}, \mathcal{O}_{i}}^{P_{0} Q_{i}}\left(t, \tau_{1}, \ldots, \tau_{i},\left(x_{0}, x_{c 0}\right)\right)$ the $\left(j_{1}, \ldots, j_{i}\right)$-th kernel of the compensated plant $P \circ Q$. Since $w_{j_{1} \ldots i_{1}}^{M}$ depends on the initial state $x_{0}^{M}$ of $M$ and $w_{j, \cdots, j_{i}}^{P \circ}$ on the initial state $\left(x_{0}, x_{c 0}\right)$ of $P \circ Q$, when imposing the coincidence between these kernels one must specify how $x_{0}^{M}$ and $\left(x_{0}, x_{c 0}\right)$ are chosen. Depending on this choice, one may formulate different matching problems. The most usual definition of the model matching problem, due to $\mathrm{Di}$ Benedetto and Isidori (1986), is given below.

Definition 1. Nonlinear model matching problem (MMP). Given a plant $P=(f, g, h)$, a model $M=\left(f^{M}, g^{M}, h^{M}\right)$ and a point $\left(x_{0}, x_{0}^{M}\right) \in$ $\mathscr{Z} \times \mathscr{X}^{M} \subset \mathbb{R}^{n} \times \mathbb{R}^{n_{M}}$, find neighbourhoods $U$ of $x_{0}$ and $U^{M}$ of $x_{0}^{M}$, an integer $v$, an open subset $V$ of $\mathscr{X}_{c} \subset \mathbb{R}^{\nu}$, a compensator $Q=(a, b, c, d)$ with $a, b, c, d$ real analytic functions defined on $V \times U$, a map $F: U \times U^{M} \mapsto V$, with the property that

$$
\begin{aligned}
w_{j_{1} \cdots j_{i}}^{P_{P} Q_{i}}\left(t, \tau_{1}, \ldots,\right. & \left.\tau_{i},\left(x, F\left(x, x^{M}\right)\right)\right) \\
& =w_{j_{1} \cdots j_{i}}^{M}\left(t, \tau_{1}, \ldots, \tau_{i}, x^{M}\right)
\end{aligned}
$$

for all $i \geq 1$, for all $1 \leq j_{i} \leq m$ and for all $\left(x, x^{M}\right) \in U \times U^{M}$. 
In Di Benedetto and Isidori (1986) an attempt has been made to generalize the solution of the linear problem, cf. Section 2, to obtain a local solution to the nonlinear MMP. This is done by defining an extended system $E$ associated with model and plant:

$$
E\left\{\begin{array}{l}
\dot{x}^{E}=f^{E}\left(x^{E}\right)+\sum_{i=1}^{m} u_{i} g_{i}^{E}\left(x^{E}\right)+\sum_{i=1}^{m} u_{i}^{M} p_{i}^{E}\left(x^{E}\right) \\
y_{i}^{E}=h_{i}^{E}\left(x_{E}\right) \quad(i \in\{1, \ldots, m\})
\end{array}\right.
$$

where $x^{E}=\left(x, x^{M}\right)$ and

$$
\begin{array}{ll}
f^{E}\left(x^{E}\right)=\left[\begin{array}{c}
f(x) \\
f^{M}\left(x^{M}\right)
\end{array}\right], & g_{i}^{E}\left(x^{E}\right)=\left[\begin{array}{c}
g_{i}(x) \\
0
\end{array}\right] \\
p_{i}^{E}\left(x^{E}\right)=\left[\begin{array}{c}
0 \\
g_{i}^{M}\left(x^{M}\right)
\end{array}\right], & h_{i}^{E}\left(x^{E}\right)=h_{i}(x)-h_{i}^{M}\left(x^{M}\right)
\end{array}
$$

Define:

$$
\begin{aligned}
\mathscr{g}^{E} & =\operatorname{span}\left\{g_{1}^{E}, \ldots, g_{m}^{E}\right\} \\
\mathscr{P}^{E} & =\operatorname{span}\left\{p_{1}^{E}, \ldots, p_{m}^{E}\right\}
\end{aligned}
$$

Theorem 3. (Di Benedetto and Isidori, 1986). The following equivalent conditions are sufficient for local solvability of MMP.

$$
\text { 1. } \mathscr{P}^{E} \subset \Delta^{*}+\mathscr{G}^{E}
$$

where $\Delta^{*}$ is the maximal $\left(f^{E}, g^{E}\right)$-invariant distribution contained in $\operatorname{Ker} \mathrm{d} h^{E}$.

2. The structure at infinity of the extended system (11) (w.r.t. the inputs $u_{1}, \ldots, u_{m}$ ) and that of the plant (6) are the same.

Now define relative degrees $\rho_{1}(x), \ldots, \rho_{m}(x)$ for the plant $P$

$$
\begin{aligned}
& \rho_{i}(x)=\inf \left\{k \in \mathbb{N} \text { s.t. } \mathscr{L}_{g_{j}} \mathscr{L}_{f}^{k-1} h_{i}(x)\right. \\
&\neq 0 \text { for some } j\} .
\end{aligned}
$$

In the same way we define relative degrees $\rho_{i}^{M}\left(x^{M}\right)$ for the model $M, \rho_{i}^{P^{\circ} Q}\left(x, x_{c}\right)$ for the compensated plant $P \circ Q$, and $\rho_{i}^{E}\left(x^{E}\right)$ for the extended system $E$.

The following will be a standing assumption throughout the paper.

(A1) All relative degrees defined above are finite and constant on an open and dense submanifold, i.e. $\rho_{i}(x)=\rho_{i}<\infty, \rho_{i}^{M}\left(x^{M}\right)=\rho_{i}^{M}<$ $\infty, \rho_{i}^{P \circ Q}\left(x, x_{c}\right)=\rho_{i}^{P \bullet Q}<\infty, \rho_{i}^{E}\left(x^{E}\right)=\rho_{i}^{E}<\infty$ on an open and dense submanifold.

Lemma 1. Let $Q$ locally solve MMP for $(M, P)$. Then $\rho_{i}^{P \circ Q}=\rho_{i}^{M}$.

Proof. Let $P \circ Q$ be given by

$$
\left\{\begin{array}{l}
\dot{\tilde{x}}=\tilde{f}(\tilde{x})+\tilde{g}(\tilde{x}) u^{M} \\
\tilde{y}=\tilde{h}(\tilde{x})=h(x)
\end{array}\right.
$$

where $\tilde{x} \in \mathscr{X} \times \mathscr{X}_{c}$. The fact that $Q$ solves MMP for $(M, P)$ implies that $u^{M}$ does not influence the outputs $h_{i}^{E}\left(x^{E}\right)=h_{i}(x)-h_{i}^{M}\left(x^{M}\right)$ of the extended system $E$, i.e. we have solved a disturbance decoupling problem. By analyticity of the data this implies that for all $k \in \mathbb{N}$ and for all $i, j \in\{1, \ldots, m\}: \mathscr{L}_{\bar{B}_{i}} \mathscr{L}_{f}^{k} \bar{h}_{i}(\bar{x})=\mathscr{L}_{g_{i}^{M}} \mathscr{L}_{f}^{k} h_{i}^{M}\left(x^{M}\right)$ and hence in particular $\rho_{i}^{P^{\circ} Q}=\rho_{i}^{M}$.

The decoupling matrix $A(x)$ of $P$ is defined on the open and dense submanifold where (A1) is satisfied in the following way:

$$
\begin{aligned}
(A(x))_{i j}=\mathscr{L}_{g_{j}} \mathscr{L}_{f}^{\rho_{i}-1} h_{i}(x) & \\
& (i, j \in\{1, \ldots, m\}) .
\end{aligned}
$$

Similarly we define decoupling matrices $A^{M}$, $A^{P \circ Q}, A^{E}$ for $M, P \circ Q, E$ respectively.

Theorem 4. If the decoupling matrix $A(x)$ of $P$ has full rank for all $x$ on an open and dense submanifold of $\mathscr{X}$, then MMP is locally solvable if and only if

$$
\rho_{i} \leq \rho_{i}^{M} \text { for all } i \in\{1, \ldots, m\} .
$$

Moreover, in this case (18) is equivalent to the conditions in Theorem 3.

Proof. See Di Benedetto and Isidori (1986) and Huijberts (1989a).

Next we formulate our main result on the MMP, of which the proof will be given in Section 5.

Theorem 5. Consider an analytic plant $P$ and an analytic model $M$. Let $x_{0}$ and $x_{0}^{M}$ be equilibrium points of, respectively, $P$ and $M$. Assume the decoupling matrix of the model has full rank around $x_{0}^{M}$. Let $L P, L M$ denote the linearizations of $P$ around $x_{0}$ and $M$ around $x_{0}^{M}$ respectively. Then under generic conditions on $P$ the MMP is locally solvable for $(M, P)$ if and only if it is solvable for $(L M, L P)$.

Roughly phrased, the theorem says that the MMP is "almost always" solvable for $(M, P)$ provided the problem is solvable for the linearization $(L M, L P)$. We refer to e.g. Tchoń (1986) for a precise mathematical definition of a generic property, and we will identify checkable and verifiable conditions on $P$ for which the above result holds true. These conditions naturally appear in the solution of the input-output decoupling problem for the plant $P$. This problem will be discussed in the next section.

Notice that Theorem 5 in principle only 
addresses the MMP in a neighbourhood of the equilibria $x_{0}$ and $x_{0}^{M}$. However, it is rather straightforward to see that the analyticity of $P$ and $M$ imply that whenever a local solution of the MMP exists around $\left(x_{0}, x_{0}^{M}\right)$, then there exists a local solution around points in an open and dense submanifold of $\mathscr{X} \times \mathscr{X}^{M}$. The theory developed in the following sections can be directly extended to this situation.

\section{THE INPUT-OUTPUT DECOUPLING PROBLEM}

We consider a nonlinear system (6). Then the (dynamic) input-output decoupling problem is defined as follows

\section{Definition 1. Input-output decoupling prob-} lem. Find (if possible) an integer $v, a$ compensator of the form (8) for (6) and an initial state $x_{c 0} \in \mathbb{R}^{v}$ for the compensator such that the overall system is input-output decoupled. That is, each of the new inputs influences one and only one of the outputs.

It is well known [cf. Isidori (1985b)] that the input-output decoupling problem for analytic systems is locally solvable by static state feedback (i.e. we can take $v=0$ ) if and only if the decoupling matrix of (6) has full rank for all $x$. The problem of dynamic input-output decoupling was studied by Singh $(1980,1981)$ via a generalization of Hirschorn's nonlinear version (Hirschorn, 1979) of the Silverman structure algorithm (Silverman, 1969). In Descusse and Moog (1985) an interesting extension to strongly left-invertible nonlinear systems of linear dynamic decoupling, as was used in Wang (1970), was given. A more transparent algorithm for the input-output decoupling problem was given in Nijmeijer and Respondek (1988), thereby generalizing and expanding the algorithm of Descusse and Moog (1985). Essentially this algorithm (as well as the others) works on an open and dense submanifold of points $\left(x, x_{c}\right)$ in $\mathscr{X} \times \mathscr{X}_{c}$. Our basic assumption will be that given the equilibrium point $x_{0}$ for $P$ there exists an equilibrium point $\left(x_{0}, x_{c 0}\right)$ for the precompensated system $P \circ Q$ at which the algorithm can be effectively applied. To make this more transparent we shortly discuss the first step of the algorithm of Nijmeijer and Respondek (1988). Given the plant $P$ we compute its decoupling matrix $A(x)$ according to equation (17). Clearly the rank of $A(x)$ is constant on an open and dense submanifold $\mathscr{X}^{1}$ of $\mathscr{Z}$. Assume $x_{0} \in \mathscr{X}^{1}$. Next a regular static state feedback.

$$
u=\alpha(x)+\beta(x) \tilde{u}
$$

is applied to $P$, and which leaves the equilibrium point $x_{0}$ invariant. Note that applying such a feedback does not change the rank of the decoupling matrix $A(x)$. The first step of the algorithm proceeds with adding a bank of integrators

$$
\left\{\begin{array}{c}
\dot{z}_{i 1}=z_{i 2} \\
\vdots \\
\dot{z}_{i k_{i}}=v_{i} \\
\bar{u}_{i}=z_{i 1}
\end{array}\right.
$$

for $i=1, \ldots, m$ and certain integers $k_{i} \in \mathbb{N}$. Note that for some indices $i, k_{i}=0$, which shows that some of the inputs $\bar{u}$ are not integrated. Obviously, adding (19), (20) to $P$ yields a system on $\mathscr{Z} \times \mathscr{Z}$, where $\mathscr{Z}=\left(z_{11}, \ldots, z_{1 k_{1}}, \ldots, z_{m 1}\right.$, $\ldots, z_{m k_{m}}$ ) and this system possesses an equilibrium point $\left(x_{0}, z_{0}\right) \in \mathscr{Z} \times \mathscr{Z}$. We now compute the decoupling matrix of $P$ together with (19), (20), to be denoted as $A(x, z)$. Again this decoupling matrix has constant rank on an open and dense submanifold $(\mathscr{X} \times \mathscr{X})^{1}$ of $\mathscr{X} \times \mathscr{X}$. We assume $\left(x_{0}, z_{0}\right)$ belongs to $(\mathscr{X} \times \mathscr{Z})^{1}$. The algorithm of Nijmeijer and Respondek (1988) now repeats the above steps of applying state feedbacks (19) and adding a bank of integrators as in (20). Moreover the algorithm terminates after a finite number of steps. Our basic assumption can now be stated as follows:

(A2) Consider the system (6) around the equilibrium point $x_{0} \in \mathscr{X}$. Then we assume that at each step of the above algorithm the decoupling matrix $A(x, z)$ has constant rank in a neighbourhood of the equilibrium point $\left(x_{0}, z_{0}\right)$.

Essentially (A2) says that the algorithm of Nijmeijer and Respondek (1988) can be applied on a neighbourhood of the equilibrium point $x_{0}$. Given the analyticity of (6) this condition is generically met for vector fields $f, g_{1}, \ldots, g_{m}$ and functions $h_{1}, \ldots, h_{m}$.

Remark 1 . Strictly speaking, the equilibria of a vector field $f(x, u)$ are all $\left(x_{0}, u_{0}\right)$ satisfying $f\left(x_{0}, u_{0}\right)=0$, whereas in assumption (A2) we restrict ourselves to equilibria of the form $\left(x_{0}, 0\right)$, i.e. we let $u_{0}=0$. If we do not restrict ourselves to the case that $u_{0}=0$ the set of equilibrium points obviously gets larger. This freedom will be explored in Example 6.2 where it is shown that the conditions for solvability of MMP are met around an equilibrium $\left(x_{0}, u_{0}\right)$ where $u_{0} \neq 0$, while the conditions are not met around an equilibrium of the form $\left(x_{0}, 0\right)$. Note however that an equilibrium of the form $\left(x_{0}, u_{0}\right)$ where $u_{0} \neq 0$ can be transformed into an equilibrium of the form $\left(x_{0}, 0\right)$ by aplying a preliminary feedback.

The following proposition shows the importance of (A2) in that under this assumption the 
solvability of the input-output decoupling problem for (6) around $x_{0}$ is completely equivalent to the solvability of the input-output decoupling problem for its linearization around $x_{0}$.

Proposition 1. Consider a nonlinear system (6). Let $x_{0}$ be an equilibrium point of the system that satisfies (A2). Then the input-output decoupling problem is solvable for (6) if and only if it is solvable for the linearization of (6) around $x_{0}$.

\section{Proof. (Necessity)}

Assume that the input-output decoupling problem is solvable for (6) by means of a compensator of the form (8). Let $x_{c 0}$ be such that $\left(x_{0}, x_{c 0}\right)$ is an equilibrium point of $P \circ Q$. Since the compensator solves the input-output decoupling problem and (A2) holds, the decoupling matrix of $P \circ Q$ has full rank at $\left(x_{0}, x_{c 0}\right)$. This also implies that the decoupling matrix of the linearization of $P \circ Q$ around $\left(x_{0}, x_{c 0}\right)$ has full rank [see e.g. Gras and Nijmeijer (1989)]. Observe that the linearization of $P \circ Q$ around $\left(x_{0}, x_{c 0}\right)$ is nothing else but the linearization of $P \circ Q$ around $x_{0}$ compensated by the linearization of $(8)$ around $\left(x_{0}, x_{c 0}\right)$. Hence the input-output decoupling problem is also solvable for the linearization of $P$ around $x_{0}$.

(Sufficiency)

Assume that the input-output decoupling problem is solvable for the linearization of (6) around $x_{0}$. It is easy to see that, since (A2) holds, the algorithm from Nijmeijer and Respondek (1988) can be applied at the same time to (6) and the linearization of (6) around $x_{0}$, meaning that at each round the feedback applied to the linearization is the linearization of the feedback applied to (6) and that we add the same bank of integrators to (6) and the linearization.

In the rest of this section, we will give some results on the input-output decoupling problem for linear systems. So, consider a linear system of the form (1). It is well known from the literature [see e.g. Wang (1970), Falb and Wolovich (1967)] that the input-output decoupling problem is solvable for (1) if and only if (1) is invertible [see Descusse and Moog (1985) for a generalization to nonlinear systems].

Apart from the relative degrees as defined in (15) we can also define another type of characteristic numbers for (1), called the essential orders. The notion of essential orders is based on the work by Cremer (1971). Here we follow Commault et al. (1986) [for a generalization to nonlinear systems, see Glumineau and Moog, (1989)].
Definition 2. (Cremer, (1971). Given a matrix $W$, the $i$ th row $w_{i}$ of $W$ is called essential if $w_{i}$ cannot be represented by a linear combination of other rows of $W$.

Now let us denote the $(\mu m, \mu m)$-matrix:

$$
\Gamma_{\mu}=\left[\begin{array}{ccccc}
C B & 0 & \cdots & \cdots & 0 \\
C A B & C B & 0 & & \vdots \\
\vdots & \vdots & \ddots & \ddots & \vdots \\
\vdots & \vdots & & \ddots & 0 \\
C A^{\mu-1} B & C A^{\mu-2} B & \cdots & \cdots & C B
\end{array}\right] .
$$

Definition 3. Consider an invertible linear system (1). Let $c_{i}$ denote the $i$ th row of $C$. The essential order of the $i$ th output of (1) is the integer $\epsilon_{i}$ defined by:

$$
\begin{array}{r}
\epsilon_{i}=\inf \left\{\mu \in \mathbb{N} \text { s.t. }\left[c_{i} A^{\mu-1} B \cdots c_{i} B 0 \cdots 0\right]\right. \\
\text { is essential in } \left.\Gamma_{n}\right\} .
\end{array}
$$

Theorem 6. Consider an invertible linear system (1). Then we have:

1. $\epsilon_{i} \geq \rho_{i}$

2. $\epsilon_{i}, \rho_{i}$ cannot be decreased by static or dynamic feedback

3. The input-output decoupling problem is solvable by static state feedback if and only if $\epsilon_{i}=\rho_{i}$

4. Among all possible decoupling compensators there is at least one for which the essential orders are not increased.

Proof. See Commault et al., 1986; Dion and Commault, 1985; Descusse et al. 1986.

Remark 2. The assertions of the above theorem also hold for nonlinear systems (6). For a proof, see Glumineau and Moog (1989), and Xia (1989).

We have as an immediate consequence (cf. Commault et al., 1986; Dion and Commault, 1985):

Corollary 1. Consider an invertible linear system (1). Then for this system there is a decoupling precompensator of minimal dimension such that the relative degrees of the decoupled system equal the essential orders of (1).

\section{PROOF OF THE MAIN THEOREM}

In this section we will first give a proof of the main theorem. After that we will make some comments on the characterization of models that can be matched starting from a specific plant. 
Assume we are given a plant $P$ with equilibrium point $x_{0}$ and a model $M$ with equilibrium point $x_{0}^{M}$ such that the decoupling matrix $A^{M}\left(x^{M}\right)$ of the model has full rank in a neighbourhood of $x_{0}^{M}$. For convenience we will first give a reformulation of our main theorem, where the generic conditions on $P$ are made explicit.

Theorem 7. Consider an analytic plant $P$ and an analytic model $M$. Let $x_{0}$ and $x_{0}^{M}$ be equilibrium points of respectively $P$ and $M$. Assume that $P$ satisfies (A2) and that the decoupling matrix of the model has full rank around $x_{0}^{M}$. Let $L P, L M$ denote the linearizations of $P$ around $x_{0}$ and $M$ around $x_{0}^{M}$ respectively. Then the MMP is locally solvable for $(M, P)$ if and only if it is solvable for $(L M, L P)$.

Before proving the main theorem we will prove some preliminaries.

Lemma 2. Let $Q$ be a compensator that locally solves the MMP for $(M, P)$, where the decoupling matrix $A^{M}\left(x^{M}\right)$ has full rank around the equilibrium $x_{0}^{M}$. Then $A^{P \circ Q}\left(x, x_{c}\right)$ has full rank on an open and dense submanifold of $\mathscr{X} \times \mathscr{X}_{c}$.

Proof. Let $P \circ Q$ be given by:

$$
\left\{\begin{array}{l}
\dot{\tilde{x}}=\tilde{f}(\bar{x})+\tilde{g}(\tilde{x}) u^{M} \\
\tilde{y}=\tilde{h}(\tilde{x})=h(x)
\end{array}\right.
$$

where $\bar{x} \in \mathscr{X} \times \mathscr{X}_{c}$. Recall from Lemma 1 that the fact that $Q$ solves MMP implies that $\rho_{i}^{P \circ Q}=\rho_{i}^{M}$. Since $Q$ solves MMP, for any $x_{*}^{M}$ there is an $\bar{x}_{*}$ such that for any $u^{M}$ and any $k \in \mathbb{N}: \mathscr{L}_{\tilde{g}} \mathscr{L}_{\tilde{f}}^{k} \bar{h}(\tilde{x})=$ $\mathscr{L}_{g^{M}} \mathscr{L}_{f^{k}}^{k} h^{M}\left(x^{M}\right)$ along the trajectories of $P \circ Q$ and $M$ starting at $\tilde{x}_{*}$ and $x_{*}^{M}$ respectively. Hence in particular there is an $\tilde{x}$ for which $A^{P^{\circ} Q}(\tilde{x})$ has full rank. Then by analyticity of the data: $A^{P \bullet Q}(\tilde{x})$ has full rank on an open and dense submanifold of $\mathscr{X} \times \mathscr{X}_{c}$.

This leads to:

Proposition 2. Consider an analytic plant $P$ and an analytic model $M$, where the decoupling matrix $A^{M}\left(x^{M}\right)$ has full rank around the equilibrium $x_{0}^{M}$. Then MMP is locally solvable for $(M, P)$ if and only if there is a dynamic compensator $Q$ such that $P \circ Q$ is decouplable by regular static state feedback and $\rho_{i}^{P \circ Q} \leq \rho_{i}^{M}$.

\section{Proof. (Necessity)}

If $Q$ locally solves MMP, then we know by Lemma 2 that $A^{P \circ Q}$ has full rank on an open and dense submanifold. Hence $P \circ Q$ is decouplable by static state feedback. Furthermore by Theorem 4: $\rho_{i}^{P \circ Q} \leq \rho_{i}^{M}$.

(Sufficiency)

Follows immediately from Theorem 4.

We now come to the proof of our main theorem:

Proof (of Theorem 7). (Necessity)

If MMP is solvable for $(M, P)$ then by Proposition 2 there is a dynamic compensator $Q$ such that $P \circ Q$ is decouplable by regular static state feedback and $\rho_{i}^{P \circ Q} \leq \rho_{i}^{M}$. Since (A2) holds, this implies that there is a compensator $L Q$ for $L P$ such that $L P \circ L Q$ is decouplable by regular static state feedback and $\rho_{i}^{L P \circ L Q} \leq \rho_{i}^{L M}$. Hence by Proposition 2, MMP is solvable for $(L M, L P)$.

\section{(Sufficiency)}

Follows immediately by reversing the reasoning of the necessity part.

Proposition 2 also enables us to characterize models that can be matched starting from a specific plant.

Theorem 8. Consider an analytic plant $P$ with equilibrium point $x_{0}$ satisfying (A2). Let the essential orders of its linearization around $x_{0}$ be denoted by $\epsilon_{i}$. Then MMP is locally solvable for $(M, P)$ where $M$ is an analytic system and the decoupling matrix $A^{M}\left(x^{M}\right)$ has full rank around the equilibrium $x_{0}^{M}$, if and only if $\epsilon_{i} \leq \rho_{i}^{M}$.

\section{Proof. (Necessity)}

Assume that MMP is solvable for $(M, P)$ where the decoupling matrix $A^{M}\left(x^{M}\right)$ has full rank around the equilibrium $x_{0}^{M}$. Then, by Theorem $7 \mathrm{MMP}$ is also solvable for $(L M, L P)$. By Proposition 2 this implies that there is a dynamic compensator $L Q$ such that $L P \circ L Q$ is decouplable by regular static state feedback and $\rho_{i}^{L P \circ L Q} \leq \rho_{i}^{L M}$. By Corollary 1 we have $\rho_{i}^{L P \circ L Q} \geq$ $\epsilon_{i}$ and thus: $\epsilon_{i} \leq \rho_{i}^{M}$.

\section{(Sufficiency)}

Consider a model $M$ for which the decoupling matrix $A^{M}\left(x^{M}\right)$ has full rank around the equilibrium $x_{0}^{M}$ and that satisfies $\epsilon_{i} \leq \rho_{i}^{M}$. By Corollary 1 there is a decoupling compensator $L Q$ for $L P$ such that the relative degrees of $L P \circ L Q$ are just $\epsilon_{i}$. By Proposition 1 (or rather the proof of it) this implies that there is a decoupling compensator $Q$ for $P$ such that the relative degrees of $P \circ Q$ also equal $\epsilon_{i}$. Thus there is a decoupling compensator $Q$ for $P$ such that $\rho_{i}^{P^{\circ} Q}=\epsilon_{i} \leq \rho_{i}^{M}$ and hence by Proposition 2 we find that the MMP is solvable for $(M, P)$. 


\section{EXAMPLES}

Example 1. We first consider the example also studied in Di Benedetto and Isidori (1986) and Di Benedetto (1988a). Here the plant is of the form

$$
P\left\{\begin{array}{l}
\dot{x}=f(x)+g_{1}(x) u_{1}+g_{2}(x) u_{2} \\
y_{i}=h_{i}(x)(i=1,2)
\end{array}\right.
$$

where

$$
\begin{aligned}
& f(x)=\left[\begin{array}{c}
0 \\
x_{4} \\
0 \\
0
\end{array}\right] \quad g_{1}(x)=\left[\begin{array}{l}
x_{3} \\
0 \\
1 \\
0
\end{array}\right] \quad g_{2}(x)=\left[\begin{array}{l}
0 \\
1 \\
1 \\
1
\end{array}\right] . \\
& h_{1}(x)=x_{2}-x_{3} \\
& h_{2}(x)=x_{1} .
\end{aligned}
$$

The relative degrees of the system equal 1 and 1 on an open and dense submanifold containing the equilibrium $\left(x_{0}, u_{0}\right)$ where $x_{0}=(0,1,1,0)$, $u_{0}=(0,0)$, and the decoupling matrix on this submanifold is given by

$$
A(x)=\left[\begin{array}{cc}
-1 & 0 \\
x_{3} & 0
\end{array}\right]
$$

It can easily be checked that (A2) is satisfied around the equilibrium point $x_{0}$. The linearization of $P$ around $x_{0}$ is given by

where

$$
L P\left\{\begin{array}{l}
\dot{x}=F x+G u \\
y=H x
\end{array}\right.
$$

$$
\begin{gathered}
F=\left[\begin{array}{llll}
0 & 0 & 0 & 0 \\
0 & 0 & 0 & 1 \\
0 & 0 & 0 & 0 \\
0 & 0 & 0 & 0
\end{array}\right] \quad G=\left[\begin{array}{ll}
1 & 0 \\
0 & 1 \\
1 & 1 \\
0 & 1
\end{array}\right] \\
H=\left[\begin{array}{rrrr}
0 & 1 & -1 & 0 \\
1 & 0 & 0 & 0
\end{array}\right] .
\end{gathered}
$$

The essential orders of $L P$ equal 2 and 2 , so in principle any two-input-two-output system that is decouplable by static feedback and has relative degrees at least equal to 2 and 2 , can be taken as a model. In particular we can take the linear model that was proposed by Di Benedetto and Isidori (1986) [see also Di Benedetto (1988a)] and is given by

where

$$
M\left\{\begin{array}{l}
\dot{x}^{M}=A^{M} x^{M}+B^{M} u^{M} \\
y^{M}=C^{M} x^{M}
\end{array}\right.
$$

$$
\begin{aligned}
A^{M} & =\left[\begin{array}{llll}
0 & 1 & 0 & 0 \\
0 & 0 & 0 & 0 \\
0 & 0 & 0 & 1 \\
0 & 0 & 0 & 0
\end{array}\right] B^{M}=\left[\begin{array}{ll}
0 & 0 \\
1 & 0 \\
0 & 0 \\
0 & 1
\end{array}\right] \\
C^{M} & =\left[\begin{array}{llll}
1 & 0 & 0 & 0 \\
0 & 0 & 1 & 0
\end{array}\right] .
\end{aligned}
$$

Applying the algorithm of Xia and Gao (1988) yields the following minimal order decoupling compensator for the plant:

$$
\left\{\begin{array}{l}
\dot{x}_{c}=v_{1} \\
u_{1}=x_{4}-x_{c} \\
u_{2}=v_{2}
\end{array}\right.
$$

where $v_{1}, v_{2}$ are the new inputs. Differentiation of the outputs of $P$ after we have applied (31) yields:

$$
\left[\begin{array}{l}
\ddot{y}_{1} \\
\ddot{y}_{2}
\end{array}\right]=\left[\begin{array}{c}
0 \\
\left(x_{4}-x_{c}\right)^{2}
\end{array}\right]+\left[\begin{array}{cc}
1 & 0 \\
-x_{3} & x_{3}+x_{4}-x_{c}
\end{array}\right]\left[\begin{array}{l}
v_{1} \\
v_{2}
\end{array}\right] \text {. }
$$

Differentiation of the outputs of the model yields

$$
\ddot{y}_{i}^{M}=u_{i}^{M} \quad(i=1,2) .
$$

Thus, MMP is solved for $(M, P)$ if we take

$$
\begin{aligned}
{\left[\begin{array}{l}
v_{1} \\
v_{2}
\end{array}\right] } & =\left[\begin{array}{c}
0 \\
-\frac{\left(x_{4}-x_{c}\right)^{2}}{x_{3}+x_{4}-x_{c}}
\end{array}\right] \\
& +\left[\begin{array}{cc}
1 & 0 \\
\frac{x_{3}}{x_{3}+x_{4}-x_{c}} & \frac{1}{x_{3}+x_{4}-x_{c}}
\end{array}\right]\left[\begin{array}{l}
u_{1}^{M} \\
u_{2}^{M}
\end{array}\right] .
\end{aligned}
$$

Combining (31) and (34), we obtain as a compensator that solves MMP:

$$
Q\left\{\begin{array}{l}
\dot{x}_{c}=u_{1}^{M} \\
u_{1}=x_{4}-x_{c} \\
u_{2}=-\frac{\left(x_{4}-x_{c}\right)^{2}}{x_{3}+x_{4}-x_{c}}+\frac{x_{3}}{x_{3}+x_{4}-x_{c}} u_{1}^{M} \\
\quad+\frac{1}{x_{2}+x_{4}-x_{c} u_{2}^{M}}
\end{array}\right.
$$

which is exactly the compensator obtained in another fashion by Di Benedetto (1988a). Also, an easy computation shows that this compensator is equivalent to the one obtained in $\mathrm{Di}$ Benedetto and Isidori (1986). Hence, using our method we have produced in a constructive way the compensator that was derived heuristically in $\mathrm{Di}$ Benedetto and Isidori (1986) and $\mathrm{Di}$ Benedetto (1988a).

Example 2. In this example our plant will be a voltage frequency controlled induction motor as described by De Luca and Ulivi (1988). As state variables we take the projections of the stator current and flux vectors on a reference frame $(\alpha, \beta)$ which is fixed to the stator windings, and the angular position of the voltage input vector. As inputs we will take the amplitude of the voltage input vector and the voltage supply frequency. The parameters $R_{\mathrm{s}}$ and $R_{\mathrm{r}}$ are the stator and rotor resistances, $L_{\mathrm{s}}$ and $L_{\mathrm{r}}$ are the 
stator and rotor self-inductances and $M$ is the mutual inductance. The speed $\omega$ can be considered as a slowly varying parameter, due to the large separation of time-scales between the mechanical and the electromagnetic dynamics. In the sequel we will assume it to be constant.

Defining $\bar{x}=\left(x_{1}, \ldots, x_{4}\right)$ and $x=\left(\bar{x}, x_{5}\right)$, the state equations are written as

$$
\dot{x}=\left[\begin{array}{c}
A \bar{x} \\
0
\end{array}\right]+\left[\begin{array}{cc}
g_{1}\left(x_{5}\right) & 0 \\
0 & 1
\end{array}\right]\left[\begin{array}{l}
u_{1} \\
u_{2}
\end{array}\right]
$$

where

$$
\begin{gathered}
A=\left[\begin{array}{cccc}
-(\alpha+\beta) & -\omega & \frac{\beta}{L_{\mathrm{s}}} & \frac{\omega}{\sigma L_{\mathrm{s}}} \\
\omega & -(\alpha+\beta) & -\frac{\omega}{\sigma L_{\mathrm{s}}} & \frac{\beta}{L_{\mathrm{s}}} \\
-\alpha \sigma L_{\mathrm{s}} & 0 & 0 & 0 \\
0 & -\alpha \sigma L_{\mathrm{s}} & 0 & 0
\end{array}\right] \\
g_{1}\left(x_{5}\right)=\left[\begin{array}{c}
\frac{\cos x_{5}}{\sigma L_{\mathrm{s}}} \\
\frac{\sin x_{5}}{\sigma L_{\mathrm{s}}} \\
\cos x_{5} \\
\sin x_{5}
\end{array}\right]
\end{gathered}
$$

and

$$
\alpha=\frac{R_{\mathrm{s}}}{\sigma L_{\mathrm{s}}}, \quad \beta=\frac{R_{\mathrm{r}}}{\sigma L_{\mathrm{r}}}, \quad \sigma=1-\frac{M^{2}}{L_{\mathrm{s}} L_{\mathrm{r}}} .
$$

Suitable outputs for the system are defined in terms of the stator fiux and the torque. Hence, the following nonlinear output functions will be used

$$
\begin{aligned}
& h_{1}(x)=\Phi_{\mathrm{s}}^{2}=x_{3}^{2}+x_{4}^{2} \\
& h_{2}(x)=T_{m}=x_{2} x_{3}-x_{1} x_{4} .
\end{aligned}
$$

If we look for equilibria of the form $\left(x_{0}, 0\right)$ (cf. Remark 1), we find $x_{0}=\left(\bar{x}, x_{5}\right)=\left(0, x_{5}\right)$, since $A$ is invertible. In these equilibria the relative degrees are infinite, so (A2) does not hold in these equilibria. It is easily seen that with

$$
\begin{aligned}
x_{0}=\left(-\frac{1}{\alpha \sigma L_{\mathrm{s}}}, 0,-\frac{\beta^{2} \sigma+\omega^{2}}{\alpha\left(\beta^{2} \sigma^{2}+\omega^{2}\right)},\right. \\
\left.\frac{\beta \omega(\sigma-1)}{\alpha\left(\beta^{2} \sigma^{2}+\omega^{2}\right)}, 0\right),
\end{aligned}
$$

$u_{0}=(1,0), \quad\left(x_{0}, u_{0}\right)$ is an equilibrium. This equilibrium can be transformed into an equilibrium of the form $\left(x_{0}, v_{0}\right)$ with $v_{0}=0$ by introducing the preliminary feedback

$$
\left[\begin{array}{l}
u_{1} \\
u_{2}
\end{array}\right]=\left[\begin{array}{l}
1 \\
x_{5}
\end{array}\right]+\left[\begin{array}{l}
v_{1} \\
v_{2}
\end{array}\right]
$$

The relative degrees of the system after we have applied the preliminary feedback equal 1 and 1 on an open and dense submanifold containing $x_{0}$, provided $M \neq 0$ and $M^{2} \neq$ $\frac{L_{\mathrm{r}}^{2} \omega^{2}-R_{\mathrm{r}}^{2}}{L_{\mathrm{r}} \omega^{2}} L_{\mathrm{s}}$, and the decoupling matrix on this submanifold is given by

$$
\begin{aligned}
& A(x)= \\
& {\left[\begin{array}{cc}
2 x_{3} \cos x_{5}+2 x_{4} \sin x_{5} & 0 \\
\left(\frac{x_{3}}{\sigma L_{\mathrm{s}}}-x_{1}\right) \sin x_{5}-\left(\frac{x_{4}}{\sigma L_{\mathrm{s}}}-x_{2}\right) \cos x_{5} & 0
\end{array}\right] .}
\end{aligned}
$$

A straightforward calculation, using REDUCE shows that (A2) is satisfied around the equilibrium point $x_{0}$. The linearization of the system around $x_{0}$ is given by

$$
L P\left\{\begin{array}{l}
\dot{x}=F x+G v \\
y=H x
\end{array}\right.
$$

where

$$
\begin{gathered}
F=\left[\begin{array}{ccccc}
-(\alpha+\beta) & -\omega & \frac{\beta}{L_{\mathrm{s}}} & \frac{\omega}{\sigma L_{\mathrm{s}}} & 0 \\
\omega & -(\alpha+\beta) & -\frac{\omega}{\sigma L_{\mathrm{s}}} & \frac{\beta}{L_{\mathrm{s}}} & \frac{1}{\sigma L_{\mathrm{s}}} \\
-\alpha \sigma L_{\mathrm{s}} & 0 & 0 & 0 & 0 \\
0 & -\alpha \sigma L_{\mathrm{s}} & 0 & 0 & 1 \\
0 & 0 & 0 & 0 & 1
\end{array}\right] \\
G=\left[\begin{array}{cc}
\frac{1}{\sigma L_{\mathrm{s}}} & 0 \\
0 & 0 \\
1 & 0 \\
0 & 0 \\
0 & 1
\end{array}\right]
\end{gathered}
$$

$$
H=\left[\begin{array}{ccc}
0 & 0 \\
-\frac{\beta \omega(\sigma-1)}{\alpha\left(\beta^{2} \sigma^{2}+\omega^{2}\right)} & -\frac{\beta^{2} \sigma+\omega^{2}}{\alpha\left(\beta^{2} \sigma^{2}+\omega^{2}\right)} & \\
\frac{-2 \beta^{2} \sigma+\omega^{2}}{\alpha\left(\beta^{2} \sigma^{2}+\omega^{2}\right)} & 2 \frac{\beta \omega(\sigma-1)}{\alpha\left(\beta^{2} \sigma^{2}+\omega^{2}\right)} & 0 \\
0 & \frac{1}{\alpha \sigma L_{\mathrm{s}}} & 0
\end{array}\right] .
$$

It can easily be checked that the essential orders of $L P$ equal 2 and 2 . Hence any two-input-two-output system that is decouplable by static state feedback and has relative degrees at least equal to 2 and 2 can be taken as a model. Using REDUCE, compensators have been calculated for several models. The results have been omitted here, because of their lengthiness. We refer to e.g. Huijberts (1989b) for more worked examples. 


\section{CONCLUSIONS}

A local solution to the nonlinear model matching problem in case the model is decouplable by static state feedback has been given. The method to obtain this solution is completely analytic.

An implication of the method that is proposed in this paper is that if we start from an equilibrium point of $P \circ Q$ that satisfies (A2) and from an equilibrium point of the model, we in fact have perfect matching: also the zero-th order kernels of the Volterra expansions of $P \circ Q$ and $M$ coincide.

It should be noted also that in this paper results from Isidori (1985a) are generalized for a linear model that is decouplable by static state feedback.

A problem that remains unsolved is the model matching problem for models that are not decouplable by static state feedback. In this case the method of this paper does give some necessary conditions: the number of inputoutput channels of $P$ that is decouplable by dynamic state feedback must be greater than or equal to the number of input-output channels of $M$ that is decouplable by static state feedback. Further research is required to obtain necessary and sufficient conditions in this case.

Another problem that remains unsolved is the problem of internal stability of the compensated plant after we have solved the nonlinear MMP. Up till now, this problem has only been addressed by Byrnes et al. (1988) in the case where the plant is a SISO-system and by Huijberts (1989a), in the case where the plant is decouplable by static state feedback. The problem consists in the fact that, even if we start from an internally stable plant and an internally stable model, we may very well introduce unobservable unstable modes in the closed loop. In order to solve this problem, further investigation of the structure of a model matching configuration is needed, especially concerning the "fixed" and "free" modes of such a configuration. For linear systems this investigation has already been performed in Morse (1973). For nonlinear systems this question is undoubtedly much more difficult. So far, only results about "fixed" modes in the solution of the input-output decoupling problem have been obtained in Isidori and Grizzle (1988). It is not clear if a similar analysis is applicable for the here considered nonlinear MMP. We leave this open for future research.

Acknowledgments-We would like to thank C. Moog and $X .-H$. Xia for sending us the preprints of their very interesting papers on minimal dynamic precompensators.

\section{REFERENCES}

Byrnes, C. I., R. Castro and A. Isidori (1988). Linear model matching with prescribed tracking error and internal stability for nonlinear systems. In A. Bensoussan and J. L. Lions (Eds), Analysis and Optimization of Systems. Lecture Notes in Control and Information Sciences, Vol. 111, pp. 249-258. Springer, Berlin.

Commault, C., J. Descusse, J. M. Dion, J. F. Lafay and M. Malabre (1986). About new decoupling invariants: the essential orders. Int. J. Control, 44, 689-700.

Cremer M. (1971). A precompensator of minimal order for decoupling a linear multivariable system. Int. J. Control 14, 1089-1103.

De Luca, A. and G. Ulivi (1988). Dynamic decoupling of voltage frequency controlled induction motors. In $\mathrm{A}$. Bensoussan and J. L. Lions (Eds), Analysis and Optimization of Systems, Lecture Notes in Control and Information Sciences, Vol. 111, pp. 127-137. Springer, Berlin.

Descusse, J., and C. Moog (1985). Decoupling with dynamics compensation for strong invertibility affine nonlinear systems. Int. J. Control, 42, 1387-1398

Descusse, J., J. F. Lafay and M. Malabre (1986). A survey on Morgan's problem. Proc. 25th Conf. on Decision and Control, Athens, pp. 1289-1294.

Di Benedetto, M. D., (1988a). A condition for the solvability of the nonlinear model matching problem. Proc. Colloque International Automatique Non Lineaire, Nantes, France.

Di Benedetto, M. D. (1988b). New results on nonlinear model matching. To appear in IEEE Trans. Aut. Control.

Di Benedetto, M. D. and A. Isidori (1986). The matching of nonlinear models via dynamic state feedback. SIAM J. Control Optimiz., 24, 1063-1075.

Dion, J. M., and C. Commault (1985). On linear dynamic state feedback decoupling. Proc. 24th Conf. on Decision and Control, Ft Lauderdale, FL, 1031-1037.

Emre, E. and M. L. J. Hautus (1980). A polynomial characterization of $(\mathrm{A}, \mathrm{B})$-invariant and reachability subspaces. SIAM J. Control Optimiz. 18, 420-436.

Falb, P. L. and W. A. Wolovich (1967). Decoupling in the design and synthesis of multivariable control systems. IEEE Trans. Aut. Control, AC-12, 651-659.

Glumineau, A. and C. H. Moog (1989). The Essential Orders and the Nonlinear Decoupling Problem. Int. J. Control, 50, 1825-1834.

Gras, L. C. J. M., and H. Nijmeijer (1989). Decoupling in nonlinear systems: from linearity to nonlinearity. IEE Proc., 136, Pt. D., 53-62.

Isidori, A. and J. W. Grizzle (1988). Fixed modes and nonlinear noninteracting control with stability. IEEE Trans. Aut. Control, AC-33, 907-914.

Hirschorn, R. M. (1979). Invertibility of nonlinear control systems. SIAM J. Control Optimiz., 17, 289-297.

Huijberts, H. J. C. (1989a). Nonlinear model matching, with an application to Hamiltonian systems. Preprints IFAC Non Linear Control Systems Design Symp. 1989, Capri.

Huijberts, H. J. C. (1989b). Nonlinear model matching: a solution and two worked examples. Proc. 1990 ACC, San Diego, pp. 155-160.

Isidori, A. (1985a). The matching of a prescribed linear input-output behavior in a nonlinear system. IEEE Trans. Aut. Control, AC-30, 258-265.

Isidori, A. (1985b). Nonlinear control systems: an introduction. Lecture Notes in Control and Information Sciences, Vol. 72. Springer, Berlin.

Malabre, M. (1982). Structure á l'infini des triplets invariants. Application à la poursuite parfaite de modèle. In A. Bensoussan and J. L. Lions (Eds), Analysis and Optimization of Systems, Lecture Notes in Control and Information Sciences, Vol. 44, pp. 43-53. Springer, Berlin.

Moog, C. H., A. M. Perdon and G. Conte (1989). Model matching and factorization for nonlinear systems: a structural approach. To appear in SIAM J. Control Optimiz. 
Moore, B. C. and L. M. Silverman (1972). Model matching by state feedback and dynamic compensation. IEEE Trans. Aut. Control. AC-17, 491-497.

Morse, A. S. (1973). Structure and design of linear model following systems. IEEE Trans. Aut. Control, AC-18, 346-354.

Morse, A. S. (1976). Minimal solutions to transfer matrix equations. IEEE Trans. Aut. Control, AC-21, 131-133.

Nijmeijer, H. and W. Respondek (1988). Dynamic Input-Output Decoupling of Nonlinear Control Systems. IEEE Trans. Aut. Control, AC-33, 1065-1070.

Silverman, L. M. (1969). Inversion of multivariable linear systems. IEEE Trans. Aut. Control, AC.14, 270-276.

Singh, S. N. (1980). Decoupling of invertible nonlinear systems with state feedback and precompensation. IEEE
Trans. Aut. Control, AC-25, 1237-1239.

Singh, S. N. (1981). A modified algorithm for invertibility in nonlinear systems. IEEE Trans. Aut. Control, AC-26, 595-598.

Tchoń, K. (1986). On stable and typical properties of control systems. Scientific Papers of the Institute on Engineering Cybernetics of the Technicla University of Wroclaw, No. 71. Wydawnictwo Politechniki Wroclawskiej, Wroclaw.

Wang, S. H. (1970). Design of precompensator for decoupling problem. Electron. Lett., 6, 739-741.

Xia, X.-H. and W.-B. Gao (1988). A minimal order compensator for decoupling a nonlinear system. Preprint.

Xia, X.-H. (1989). New invariants-essential ranks of nonlinear systems. Preprint. 